\title{
AVALIAÇÃO DAS TÉCNICAS CIRÚRGICAS PARA TRATAMENTO DA MIELORRADICULOPATIA ESPONDILÓTICA CERVICAL
}

\author{
EVALUATION OF SURGICAL TECHNIQUES FOR TREATMENT OF CERVICAL \\ SPONDYLOTIC MYELORADICULOPATHY \\ EVALUACIÓN DE LAS TÉCNICAS QUIRÚRGICAS PARA EL TRATAMIENTO DE LA \\ MIELORRADICULOPATIIA CERVICAL ESPONDILÓTICA
}

Alexandre MeluzzI1, Mário Augusto Taricco², Roger Schimidt Brock1, Mário Rubem Pena Dias', Gilberto Nakaguawa1,

Vinícius Monteiro de Paula Guirado', Manoel Jacobsen Teixeira ${ }^{3}$

\begin{abstract}
RESUMO
Objetivo: Avaliar a eficácia do tratamento cirúrgico da mielorradiculopatia espondilótica cervical na produção de melhora neurológica pós-operatória, aferida em pontos pela escala da JOA e taxa de recuperação e as complicações do tratamento. Métodos: Análise dos prontuários e os exames de imagem de 200 indivíduos submetidos a tratamento cirúrgico da mielorradiculopatia cervical no HC-FMUSP, no período de janeiro de 1993 a janeiro de 2007. A avaliação clínica foi quantificada pela escala da JOA, com média de segmento de 06 anos e 08 meses. Resultados: Evidenciou-se melhora neurológica pós-operatória nas vias anterior e posterior, exceto nas laminectomias sem fusão, onde houve piora neurológica tardia. A via anterior mostrou um significante maior índice de complicações, relacionados a déficit de fusão intervertebral, deslocamento de enxerto, síndrome de disco adjacente, disfonia, disfagia, o mau posicionamento de enxerto e placas, lesão de raiz nervosa e significativo maior índice de re-intervenção cirúrgica. Na via posterior maior ocorrência de instabilidade em cifose pós-operatória na laminectomia, não sendo observada na laminoplastia, esta última com índices semelhantes aos encontrados na via anterior. Não houve melhora da dor axial nas laminoplastias e houve piora nas laminectomias, enquanto que nas discectomias e corpectomias houve significativa melhora do sintoma. Conclusão: As vias anterior e posterior foram eficazes em produzir melhora neurológica, exceto as laminectomias sem fusão. A via anterior produziu mais complicações, mas trata melhor a dor.
\end{abstract}

Descritores: Doenças da medula espinal/Mielopatia; Disco intervertebral; Laminectomia.

\begin{abstract}
Objective: To evaluate the efficacy of surgical treatment of cervical spondylotic myeloradiculopathy in the production of postoperative neurological improvement, measured in points by the scale of JOA (Japanese Orthopaedic Association) and the recovery rate and complications of therapy. Methods: Analysis of medical records and imaging studies of 200 patients undergoing surgical treatment of cervical myeloradiculopathy in HC-FMUSP, from January 1993 to January 2007. Clinical evaluation was quantified by the scale of the JOA, with an average follow-up of 6 years and 8 months. Results: There was post-operative neurological improvement in the anterior and posterior approach, except in laminectomy without fusion, where late neurological deterioration was observed. The anterior approach showed a significantly higher rate of complications related to poor fusion, intervertebral displacement of the graft, adjacent disc syndrome, dysphonia, dysphagia, poor positioning of the graft and plates, nerve root injury and significant higher rate of re-operation. In the posterior approach, increased occurrence of instability and kyphosis in the postoperative, in laminectomy were found, whereas in laminoplasty these conditions were not observed, presenting similar rates to those found for the anterior approach. There was no improvement in axial pain in the laminoplasties and worsening in laminectomies, whereas in discectomias and corpectomias there was significant relief of symptoms. Conclusion: The anterior and posterior routes were effective in producing neurological improvement, except for laminectomy without fusion. The anterior approach produced more complications, but is better for pain treatment.
\end{abstract}

Keywords: Diseases of the spinal cord / Myelopathy; Intervertebral disc; Laminectomy.

RESUMEN

Objetivo: Evaluar la eficacia del tratamiento quirúrgico de la mielorradiculopatía cenvical espondilótica en la producción de la mejoría neurológica posoperatoria, medida en puntos en la escala de la JOA y la tasa de recuperación y las complicaciones de la terapia. Métodos: Análisis de los registros médicos y de los exámenes de imagen de 200 pacientes sometidos a tratamiento quirúrgico de la mielorradiculopatía cervical en el HC-FMUSP, desde enero de 1993 a enero de 2007. La evaluación clínica se cuantificó mediante la escala de la JOA, con un segmento promedio de 06 años y 08 meses. Resultados: Se observó una mejoría neurológica posoperatoria en las vías anterior y posterior, con excepción de las laminectomías sin fusión, donde hubo deterioro neurológico tardío. La vía anterior mostró una tasa significativamente mayor de complicaciones relacionadas con el déficit de fusión intervertebral, desplazamiento del injerto, síndrome de disco adyacente, disfonía, disfagia, o mala posición de injerto y placas, lesión de la raíz del nervio y tasa significativamente mayor de nueva intervención quirúrgica. En la vía posterior, mayor incidencia de inestabilidad en la cifosis posoperatoria, en la laminectomía, y en la laminoplastia no se observó esto, teniendo esta última tasas similares a las encontradas en la vía anterior. No hubo mejoría en el dolor axial en las laminoplastias y se agravó en las laminectomías, mientras que en las discectomías y las corpectomías se produjo una mejoría significativa de los síntomas. Conclusión: Las vías anterior y posterior fueron eficaces en la producción de mejoría neurológica, con excepción de las laminectomías sin fusión. La vía anterior produjo más complicaciones, pero el dolor es mejor tratado.

Descriptores: Enfermedades de la médula espinal / Mielopatía; Disco intervertebral; Laminectomía.

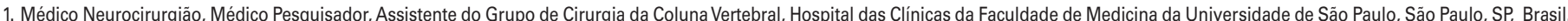

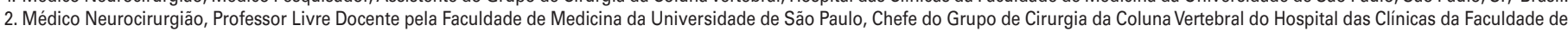
Medicina da Universidade de São Paulo, São Paulo, SP, Brasil.

3. Médico Neurocirurgião, ProfessorTitular de Neurocirurgia do Hospital das Clínicas da Faculdade de Medicina da Universidade de São Paulo, São Paulo, SP, Brasil.

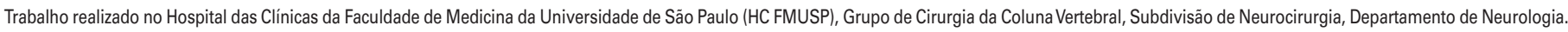
Correspondência: Av. Dr. Enéas de Carvalho Aguiar, 255 - CEP 05403-000 - São Paulo, SP. Brasil. E-mail: alemeluzzi@ig.com.br

Recebido em 30/07/2010, aceito em 19/08/2011 


\section{INTRODUÇÃO}

Avaliar a eficácia de cada tipo particular de tratamento para a mielopatia espondilótica cervical é uma tarefa difícil uma vez que os relatos de casos evidenciam que mais de $18 \%$ dos casos podem melhorar espontaneamente, 40\% podem manter-se estáveis e 40\% podem deteriorar sem nenhum tipo de tratamento ${ }^{1}$. Há certo tipo de consenso, na literatura, que pacientes pouco ou moderadamente afetados podem ser cuidadosamente observados. Para pacientes com doença em evolução algum tipo de tratamento cirúrgico é admissível ${ }^{2}$.

A cirurgia é normalmente necessária para descomprimir os elementos neurais, restaurar a lordose, e estabilizar a coluna para prevenir degeneração adicional no nível acometido ${ }^{3}$. O objetivo da cirurgia é aumentar o diâmetro ântero-posterior do canal medular, reduzindo assim a compressão sobre a medula, o que pode ser conseguido através de múltiplas técnicas, pelas vias de acesso anterior (discectomia e corpectomia) ou posterior (laminectomia e laminoplastia), cada uma com vantagens e desvantagens ${ }^{4-6}$.

A cirurgia, quando indicada neste contexto, é superior frente às medidas conservadoras, na produção de melhora neurológica ou estabilização de quadros em evolução. Fortes evidências sugerem que a precoce realização da cirurgia (sintomas com início há menos de 01 ano) é associada à substancial melhora no prognóstico neurológico ${ }^{7}$.

Além disso, múltiplos fatores prognósticos devem ser levados em consideração na decisão da indicação de algum tipo de tratamento cirúrgico, entre eles o tipo específico de patologia, a localização da lesão, o número de níveis vertebrais envolvidos, a idade no momento da cirurgia, o tempo de evolução da doença, o status neurológico pré-cirúrgico, e a presença de mielomalácia na RNM pré-operatória. Estes fatores podem ajudar a predizer a evolução operatória e podem auxiliar na escolha da técnica na abordagem cirúrgica.

A determinação da localização e a extensão da doença são fundamentais na escolha do acesso cirúrgico adequado para o tratamento da mielopatia ${ }^{8}$. Uma alteração ventral pontual é mais acessível por uma via anterior direta. Doenças limitadas ao espaço discal, como herniações de material macio, osteófitos ou complexos disco-osteofitários, são efetivamente descomprimidos por uma via anterior, com ou sem fusão e dependente de uma curvatura lordótica da coluna cervical ${ }^{9}$. Quando a doença acomete múltiplos espaços discais, e está limitada a estes, múltiplas discectomias pela via anterior são eficazes. Entretanto, quando há migração de material herniado ou quando o osteófito inclui uma porção considerável do corpo vertebral, este acesso pode não ser adequado.

Em outros casos em que a patologia se estende por múltiplos níveis vertebrais, e acomete além do espaço discal a área posterior ao corpo vertebral, a discectomia em um ou múltiplos níveis pode somente remover parcialmente a compressão. Nestes casos um acesso anterior com uma abordagem ventral extensa pode produzir uma descompressão mais desejada. Nestes casos deve ser indicado a corpectomia, em um ou mais segmentos, e fusão. Muitos autores reservam acessos anteriores extensos para doença que envolva esse limite a um ou dois níveis de corpos vertebrais ${ }^{10-12}$.

Alguns autores relatam que a doença que envolve mais de dois níveis de corpos vertebrais é tratada de uma forma mais adequada por uma via dorsal ou posterior. A área da estenose pode incorporar múltiplos níveis cervicais adjacentes ou diferentes níveis isolados ${ }^{13}$.

O processo degenerativo da espondilose normalmente inclui alterações degenerativas hipertróficas de maneira circunferencial. Facetas articulares aumentadas e espessamento do ligamento amarelo podem contribuir substancialmente para a estenose do canal. Para patologias degenerativas em múltiplos níveis e compressões posteriores é indicado o acesso posterior. Estes, incluindo a laminectomia, com ou sem fixação, e a laminoplastia provém expansão cirúrgica ao canal vertebral e descompressão pela migração da medula.

Os exames de imagem obtidos antes do procedimento cirúrgico fornecem assistência muito significativa na determinação da via e da modalidade cirúrgica a ser adequadamente empregada. Alguns autores pregam que a perda da lordose ou a evidência de cifose impõe a indução de fusão no local ou no segmento cervical, tanto na laminectomia como na descompressão pela via anterior. A curvatura mais adequada para a laminectomia sem fusão é a lordose. Muitos autores concordam que a deformidade pós-laminectomia pode ser evitada pela restrição da laminectomia somente para a coluna lordótica e pela contra-indicação desta e da laminoplastia na coluna cifótica ${ }^{9,14,15}$

O mau-alinhamento sagital pode levar a resultados pós-operatórios ruins ${ }^{15-17}$. Na maior parte dos casos, isto é uma contra-indicação para a laminectomia e para a laminoplastia, porém uma subpopulação de pacientes nestas condições, cuidadosamente selecionados, pode se beneficiar da laminoplastia. Chiba ${ }^{15}$ mostrou que a altura da coluna cervical tinha algum grau de correlação com a recuperação após a laminoplastia em pacientes com mau alinhamento sagital. A redundância medular induzida pelo encurtamento da altura da coluna cervical, causada por múltiplos níveis de degeneração discal pode aliviar a compressão da medula mesmo na presença do mau alinhamento. Na maior parte dos casos com mau alinhamento sagital e cifose a via anterior é a mais indicada, com a possibilidade de intervenção para correção, parcial, da mesma.

Estudos biomecânicos realizados em modelos animais, comparando-se a laminectomia com a laminoplastia, demonstraram um aumento na cifose radiológica pós-laminectomia, mas não na laminoplastia ou no grupo de intactos. O modelo evidenciou um aumento na instabilidade sagital no grupo pós-laminectomia. A lâmina aparentemente provém estabilidade sagital, especialmente nas extremidades da coluna cervical em $\mathrm{C} 2$ e em $\mathrm{C} 7^{18}$. A preservação da lâmina ainda pode prover uma barreira anatômica prevenindo a formação de uma cicatriz sobre a dura-máter. Yonenobu ${ }^{19}$ mostrou que uma lâmina aberta, mas retida, pode prevenir a formação de uma membrana, justa dura-máter, após a laminectomia.

A boa evolução dos casos submetidos ao acesso anterior, através do espaço discal, depende da formação de uma artrodese pós-cirúrgica estável, uma vez que a corpectomia e a discectomia, em um ou múltiplos níveis, baseiam-se na artrodese. A taxa de não união para uma fusão do tipo Smith-Robinson, não instrumentada, usando osso autólogo, é em torno de 3 a 42\%, dependendo do autor $^{10,20}$. Estudos evidenciam taxas de pseudo-artrose sintomática superiores a $70 \%{ }^{14}$

Acredita-se que a maioria das falhas de união do enxerto não é resultado de colapso do mesmo e sim na falha de uma ou das duas interfaces do enxerto em ossificar. Aumentando-se o número de níveis de discectomias aumenta-se o número de interfaces do enxerto que podem ter problemas para calcificar e unir.

Alguns autores preferem contornar este problema com a realização de corpectomias e locação de enxerto único ou pela utilização da via posterior na necessidade de segmentos extensos de cirurgia. A ocorrência de pseudo-artrose pós-cirúrgica se correlaciona com mau prognóstico pós-operatóriog.

Outra séria complicação relacionada com o uso de enxertos é o seu deslocamento pós-operatório. A incidência desta complicação tem sido relatada em taxas de ocorrência de até $29 \%$ e pode levar a sequelas neurológicas catastróficas ${ }^{9,21}$. O deslocamento do enxerto pode resultar em lesão neurológica, obstrução das vias aéreas ou lesão esofágica ${ }^{921}$. O uso de placas cervicais ventrais pode reduzir a incidência do deslocamento dos enxertos. Vaccaro ${ }^{22}$ demonstrou que corpectomias em múltiplos níveis com instrumentação anterior apresentaram taxa de 9\% de deslocamento do enxerto quando em dois níveis, e 50\% em três ou quatro níveis.

Inicialmente, nas décadas de 50 e 60, as estratégias cirúrgicas para o tratamento da mielopatia cervical foram por via posterior, usando-se laminectomias extensas ${ }^{23}$. Em pouco tempo muitas complicações pós-operatórias passaram a ser observadas ${ }^{24}$. Em modelos animais observou-se um aumento na flexibilidade da coluna cervical pós-laminectomia, mais importante nos segmentos mais inferiores, e em todos os planos de mobilização, o que foi associado clinicamente com a cifotização ${ }^{25}$.

A cifotização da coluna cervical, pós-laminectomia, é uma complicação muito conhecida em populações pediátricas. Em uma série de casos, 100\% dos pacientes com menos de 15 anos desenvol- 
veu deformidade em cifose pós-laminectomia ${ }^{26}$. Outros estudos demonstraram deterioração neurológica tardia secundária a instabilidade e cifose em adultos ${ }^{17}$. Deterioração neurológica tardia pode ainda resultar da formação de membranas pós-laminectomia. A cicatriz fibrosa que se forma sobre a dura-máter pode causar nova compressão da medula, dorsalmente, sobre o sítio da laminectomia ${ }^{19}$. A laminoplastia foi desenvolvida para resolver muitas limitações da laminectomia. No Japão havia a necessidade por uma nova técnica para descomprimir longos segmentos, em compressões extensas, causadas pela ossificação do ligamento longitudinal posterior (OLLP). Para longas extensões de compressão, exigindo longas construções com enxerto e placas, os acessos anteriores mostravam muitas complicações, incluindo deslocamento do enxerto, e pseudo-artrose. A laminectomia apresentava problemas como instabilidade e cifotização, membrana pós-laminectomia e deterioração neurológica tardia. A laminoplastia veio a ser uma solução para muitas destas possíveis complicações, com redução, já bem documentada na literatura, nas taxas de complicações pós-operatórias.

Em um estudo comparativo entre corpectomia e laminoplastia, ambas as opções foram eficazes em estabilizar ou promover alguma melhora no quadro neurológico, porém a laminoplastia mostrou necessitar de menor analgesia pós-operatória e uma menor taxa de complicações pós-operatórias ${ }^{12}$.

Alguns autores têm documentado ocorrência de dor axial após acessos posteriores. Hosono ${ }^{27}$ descreveu dor cervical e nos ombros após laminoplastia com incidência de 60\% superior ao observado no grupo submetido à corpectomia. Outros estudos na realidade demonstraram uma melhora na dor pós-operatória em pacientes submetidos à laminoplastia ${ }^{12}$.

Yoshida ${ }^{28}$ observou que $40 \%$ dos pacientes submetidos à laminoplastia apresentaram algum tipo de dor axial. Entretanto isso ocorreu quase que na totalidade em pacientes que tinham sintomas antes da cirurgia.

A aceleração da degeneração em nível adjacente é outro fator a ser considerado na escolha da via de acesso e da técnica cirúrgica. Acessos anteriores são associados com complicações decorrentes da necessidade de fusão vertebral.

A descompressão ventral e a fusão podem resultar em maior stress mecânico e aumento da amplitude de movimento, em níveis discais adjacentes ao complexo vertebral fundido, resultando em aceleração da velocidade de degeneração nestes discos, podendo levar ao surgimento de novos sintomas de mielorradiculopatia em virtude de novos quadros de compressão medular ou radicular, a denominada síndrome do disco adjacente á fusão na coluna cervical ${ }^{29}$.

Outras complicações relatadas nas corpectomias estão relacionadas com o acesso cirúrgico. Disfagia e disfomia persistentes foram observadas em $31 \%$ dos casos ${ }^{12}$.

Ainda são descritos lesões parciais ou permanentes de raízes.

Mesmo levando em consideração todos estes dados, existem ainda muitas controvérsias sobre as indicações das diversas modalidades de cirurgia para o tratamento da mielopatia espondilótica cervical. Assim propomos a análise que apresentamos.

\section{MATERIAL E MÉTODO}

Análise retrospectiva dos prontuários e exames de imagem (Radiografia simples neutra, em ântero-posterior e lateral, Radiografia dinâmica em lateral, em flexão e extensão, Ressonância magnética e eventualmente a Tomografia Computadorizada da coluna cervical), de 200 casos, com síndrome clínica de mielopatia ou mielorradiculopatia, que foram submetidos a tratamento cirúrgico da doença degenerativa da coluna cervical no Hospital das Clínicas da Faculdade de Medicina da Universidade de São Paulo, no período de março de 1993 a janeiro de 2007.

Utilizou-se a via anterior em 160 casos e a via posterior sem fusão em 40, em 133 casos a apresentação foi com quadro de mielopatia pura e em 67 casos, mielopatia associada à radiculopatia (mielorradiculopatia).

Na via anterior foi realizada a descompressão por discectomia ou a corpectomia, em um ou múltiplos níveis. Na via posterior utilizou-se a laminectomia sem fixação, a laminoplastia ou a arcocristectomia.

Excluiu-se os casos associados à artrite reumatóide, espondilite anquilosante, diabetes mellitus, alcoolismo, cirurgia prévia na coluna cervical, trauma cervical, segmento clínico inferior a 24 meses e dados incompletos no prontuário.

A avaliação clínica foi embasada no exame neurológico no momento do diagnóstico com padrão de mielopatia ou mielorradiculopatia e quantificada pela escala da JOA (Japanese Ortopaedic Association) traduzida (Tabela 1). Os pacientes foram reavaliados, utilizando-se a mesma escala, do primeiro ao sétimo dias do pós-operatório (imediato) e na última avaliação ambulatorial, com um mínimo de 24 meses (tardia) quando utilizamos também a taxa de recuperação (JOA) como referência (Tabela 2). Foram distribuídos em grupos, de acordo com a via de acesso e a modalidade cirúrgica.

A avaliação radiográfica consistiu na observação do alinhamento, presença ou não de lordose fisiológica, retificação ou inversão (cifotização) e ânterolistese e retrolistese dos níveis adjacentes à fusão. A lordose cervical foi avaliada na radiografia em perfil neutro, traçando-se uma linha perpendicular ao platô inferior de C2 e platô superior de C7, medida ainda realizada nas incidências em perfil em flexão e extensão. Nas três situações a medida entre cortical póstero-inferior da vértebra superior à cortical póstero-superior da vértebra inferior dos níveis adjacentes a artrodese. Uma listese maior que $02 \mathrm{~mm}$ no neutro ou uma mudança maior que $02 \mathrm{~mm}$ nas distâncias dinâmicas definiram instabilidade. Todos os pacientes com instabilidade no exame radiológico, estático ou dinâmico, foram submetidos à fixação interna com placa e parafusos.

A degeneração discal foi quantificada pela escala modificada de Kellgren (Tabela 3), nos períodos antes e após 24 meses da cirurgia, em todos os grupos. Esta escala classifica, na radiografia em perfil neutro, de modo progressivo, a severidade da degeneração discal, de zero ou ausente até quatro ou severa ${ }^{30}$

Tabela 1. Escala da JOA para mielopatia Cervical da Japanese Orthopaedic Association (JOA): 0 a 17 pontos.

\begin{tabular}{|c|c|c|}
\hline \multirow[t]{6}{*}{$\mathrm{I}$} & \multicolumn{2}{|l|}{ Função motora no membro superior } \\
\hline & Impossível comer com colher ou abotoar camisa & 0 \\
\hline & Possível comer com colher, impossível abotoar camisa & 1 \\
\hline & Possível abotoar camisa, com grande dificuldade & 2 \\
\hline & Possível abotoar camisa, com dificuldade & 3 \\
\hline & Normal & 4 \\
\hline \multirow[t]{6}{*}{ II } & \multicolumn{2}{|l|}{ Função motora no membro inferior } \\
\hline & Impossível & 0 \\
\hline & Necessita bengala ou auxílio em solo plano & 1 \\
\hline & Necessita auxílio em escadas & 2 \\
\hline & Anda sem auxilio, mas lentamente & 3 \\
\hline & Normal & 4 \\
\hline \multirow[t]{13}{*}{ III } & \multicolumn{2}{|l|}{ Função sensitiva } \\
\hline & MEMBRO SUPERIOR & \\
\hline & Distúrbio sensitivo aparente & 0 \\
\hline & Distúrbio sensitivo mínimo & 1 \\
\hline & Normal & 2 \\
\hline & MEMBRO INFERIOR & \\
\hline & Distúrbio sensitivo aparente & 0 \\
\hline & Distúrbio sensitivo mínimo & 1 \\
\hline & Normal & 2 \\
\hline & Tronco & \\
\hline & Distúrbio sensitivo aparente & 0 \\
\hline & Distúrbio sensitivo mínimo & 1 \\
\hline & Normal & 2 \\
\hline \multirow[t]{5}{*}{ IV } & \multicolumn{2}{|l|}{ Função vesical } \\
\hline & Retenção urinária ou incontinência & 0 \\
\hline & Sensação de retenção/perda de pequeno fluxo & 1 \\
\hline & Retenção urinária e/ou polaciúria & 2 \\
\hline & Normal & 3 \\
\hline
\end{tabular}


Tabela 2. Taxa de recuperação (JOA).

Taxa de recuperação $(\%)=($ JOA Pós-op - JOA Pré-op) X 100

$$
17 \text { - JOA Pré-op }
$$

Tabela 3. Escala modificada de Kellgren para doença degenerativa.

\begin{tabular}{c|l}
\hline Kellgren & \multicolumn{1}{|c}{ Alterações radiográficas } \\
\hline 0 & Sem alterações radiológicas \\
\hline 1 & Osteófito anterior \\
\hline 2 & Osteófito anterior, possível estenose, esclerose leve de platôs \\
\hline 3 & Osteófitos moderados, estenose, esclerose de platôs \\
\hline 4 & Osteófitos múltiplos, estenose severa, esclerose severa de platôs \\
\hline
\end{tabular}

A Ressonância magnética foi solicitada no pré-operatório em todos os casos, e novamente solicitada quando da presença de novos sintomas clínicos ou nos casos sem melhora neurológica precoce após o tratamento cirúrgico. Tardiamente foi solicitada em todos os casos. Onde havia evidências de calcificações ligamentares ou articulares a Tomografia computadorizada complementou o estudo radiológico, para fins de diagnóstico acurado e planejamento cirúrgico.

\section{RESULTADOS}

\section{Via de acesso para abordagem cirúrgica:}

Do total de 200 procedimentos 160 foram realizados por via anterior (80\%) e 40 por via posterior (20\%) (Tabela 4 e 5).

Tabela 4. Via de acesso e evolução operatória JOA.

\begin{tabular}{c|c|c|c|c}
\hline & $\mathbf{f}$ & JOA PRÉ & JOA PÓS & JOA TARDIO \\
\hline Anterior & 160 & 9,55 & 13,4 & 12,83 \\
\hline Posterior & 40 & 9,95 & 11,77 & 11,8 \\
\hline
\end{tabular}

$\mathrm{X}-\mathrm{X}^{1}=3,77 / \mathrm{X}-\mathrm{X}^{2}=3,2$

$X-X^{1}=2,14 / X-X^{2}=2,17$

$3,77 / 2,48 / 12,64=3,77 / 0,196=19,23 / / / / 3,2 / 2,48 / 12,64=2,21 / 0,196=16,32$ $2,14 / 2,48 / 6,32=2,14 / 0,39=5,48 / / / / 2,17 / 2,48 / 6,32=2,17 / 0,39=5,56$ nível de significância $\alpha=0,001$ e z crítico 3,29 / $\delta=2,48 / \mathrm{X}=9,63$

Tabela 5. Via de acesso e evolução operatória segundo a Taxa de recuperação.

\begin{tabular}{c|c|c|c}
\hline & f & TX Rec Pré & TX Rec Pós \\
\hline Anterior & 160 & 42,79 & 41,40 \\
\hline Posterior & 40 & 27,75 & 27,73 \\
\hline Geral & 200 & 39,78 & 38,65 \\
\hline
\end{tabular}

Houve melhora estatisticamente significativa para $\alpha=0,001$ no período pós operatório na pontuação da escala da JOA para os grupos abordados por via anterior e posterior. A taxa de recuperação na via anterior foi superior a da via posterior (Tabela 6).

No grupo abordado pela via posterior a média de idade está acima do limite superior do intervalo de confiaça para o grupo, assim interferindo negativamente no desempenho de melhora, uma vez que sabidamente quanto maior a idade menor a chance de melhora (Tabela 7).

No grupo abordado pela via posterior houve maior incidência de pacientes sem melhora ou com piora neurológica.

\section{Modalidades cirúrgicas}

Entre os procedimentos por via posterior 06 foram arcocristectomias (3\%), 18 laminectomias (9\%) e 16 laminoplastias (8\%). Entre os procedimentos por via anterior, 56 discectomia sem fusão e sem fixação (28\%), 42 casos discectomia com fusão e sem fixação (21\%), 28 casos discectomia com fusão e com fixação (14\%), 25 casos corpectomia em 1 nível e fusão (12,5\%), 07 corpectomia em 2 níveis e fusão (3,5\%), 02 corpectomia em 3 níveis e fusão (1\%) (Tabelas 8, 9, 10).
Tabela 6. Idade e período de evolução pré-operatória.

\begin{tabular}{l|c|c|c}
\hline & f & Idade & Períod evol pré op \\
\hline Anterior & 160 & 50,57 & 16,75 \\
\hline Posterior & 40 & 59,77 & 26,02 \\
\hline Intervalo de confiança \\
\hline SIM & $52,41 \pm 3,18(49,23-55,59)$ e 18,6 $\pm 3,95(14,65-22,55)$ \\
\hline NÃO & $52,41 \pm 6,36(46,05-58,77)$ e $18,6 \pm 7,91(10,69-26,51)$ \\
\hline
\end{tabular}

Tabela 7. Via cirúrgica e evolução operatória.

\begin{tabular}{c|c|c|c|c}
\hline & f & Melhora & Inalterado & Piora \\
\hline Anterior & 160 & $131 / 81,8 \%$ & $20 / 12,5 \%$ & $9 / 5,6 \%$ \\
\hline Posterior & 40 & $29 / 72,5 \%$ & $8 / 20 \%$ & $3 / 7,5 \%$ \\
\hline$\Sigma$ & 200 & 160 & 28 & 12 \\
\hline
\end{tabular}

\section{Via anterior}

Tabela 8. Modalidades cirúrgicas por via anterior.

\begin{tabular}{c|c|c|c}
\hline & f & fr & $\%$ \\
\hline Discectomia sem fusão e sem fixação & 56 & 0,28 & $28 \%$ \\
\hline Acrílico & 5 & 0,0312 & $3,12 \%$ \\
\hline Nada & 51 & 0,318 & $31,8 \%$ \\
\hline 1 Nível & 39 & 0,243 & $24,3 \%$ \\
\hline 2 Níveis & 16 & 0,1 & $10 \%$ \\
\hline 3 Níveis & 1 & 0,006 & $0,6 \%$ \\
\hline Discectomia com fusão e semfixação & 42 & 0,21 & 21 \\
\hline Cage & 30 & 0,187 & $18,7 \%$ \\
\hline líaco & 12 & 0,075 & $7,5 \%$ \\
\hline 1 NíVEL & 30 & 0,187 & $18,7 \%$ \\
\hline 2 NíVEIS & 10 & 0,06 & $6 \%$ \\
\hline 3 NíVEIS & 2 & 0,0125 & $1,25 \%$ \\
\hline Discectomia com fusão e com fixação & 28 & 0,14 & 14 \\
\hline Placa/Cage conjugados & 22 & 0,137 & $13,7 \%$ \\
\hline Cage & 6 & 0,037 & $3,75 \%$ \\
\hline 1 Nível & 21 & 0,131 & $13,1 \%$ \\
\hline 2 Níveis & 6 & 0,037 & $3,75 \%$ \\
\hline 3 Níveis & 1 & 0,006 & $0,6 \%$ \\
\hline Corpectomia em 1 nível & 25 & 0,125 & 12,5 \\
\hline Corpectomia em 2 níveis & 7 & 0,03 & 3,5 \\
\hline Corpectoma em 3 níveis & 2 & 0,01 & 1 \\
\hline$\Sigma$ & 160 & 0,8 & 80 \\
\hline & & &
\end{tabular}

Tabela 9. Modalidades cirúrgicas por via anterior e evolução operatória.

\begin{tabular}{c|c|c|c}
\hline & Joa pré & Joa pós & Joa tardio \\
\hline Discectomia sem fusão e sem fixação & 10 & 12,57 & 12,3 \\
\hline Discectomia com fusão e sem fixação & 9,35 & 12,78 & 12,35 \\
\hline Discectomia com fusão e com fixação & 9,14 & 13,5 & 13,57 \\
\hline Corpectomia em 1 nível & 8,92 & 11,96 & 12,28 \\
\hline Corpectomia em 2 níveis & 10,57 & 13,85 & 14,42 \\
\hline Corpectomia em 3 níveis & 11 & 15 & 15,5 \\
\hline$\sum$ Via anterior & 9,55 & 13,4 & 12,83 \\
\hline
\end{tabular}

$X-X^{1}=2,94 / X-X^{2}=2,67$

$X-X^{1}=3,15 / X-X^{2}=2,72$

$X-X^{1}=3,87 / X-X^{2}=3,94$

$X-X^{1}=2,33 / X-X^{2}=2,65$

$X-X^{1}=4,22 / X-X^{2}=4,79$

$X-X^{1}=5,37 / X-X^{2}=5,87$

$X-X^{1}=3,77 / X-X^{2}=3,2$

$\mathrm{n}=562,94 / 2,48 / 7,48=2,94 / 0,331=8,88 / / / / 2,67 / 2,48 / 7,48=2,67 / 0,331=8,06$

$\mathrm{n}=423,15 / 2,48 / 6,48=3,15 / 0,38=8,28 / / / / 2,72 / 2,48 / 6,48=2,72 / 0,38=7,15$

$\mathrm{n}=283,87 / 2,48 / 5,29=3,87 / 0,468=8,26 / / / / 3,94 / 2,48 / 5,29=3,94 / 0,468=8,41$

$n=252,33 / 2,48 / 5=2,33 / 0,496=4,69 / / / / 2,65 / 2,48 / 5=2,65 / 0,496=5,34$

$\mathrm{n}=74,22 / 2,48 / 2,64=4,22 / 0,93=4,53 / / / / 4,79 / 2,48 / 2,64=4,79 / 0,93=5,15$

$\mathrm{n}=25,37 / 2,48 / 1,41=5,37 / 1,76=3,05 / / / / 5,87 / 2,48 / 1,41=5,87 / 1,76=3,33$

$\mathrm{n}=1603,77 / 2,48 / 12,64=3,77 / 0,196=19,23 / / / / 3,2 / 2,48 / 12,64=2,21 / 0,196=11,27$ nível de significância $\alpha=0,001$ e z crítico 3,29 / $\delta=2,48 / X=9,63$. 
Tabela 10. Modalidades cirúrgicas por via anterior e evolução operatória.

\begin{tabular}{c|c|c}
\hline & TX Rec Pré & TX Rec Tar \\
\hline Discectomia & 42,65 & 39,46 \\
\hline Corpectomia & 43,31 & 48,63 \\
\hline 1 Nível & 41,70 & 44,68 \\
\hline 2 Níveis & 42,83 & 56,71 \\
\hline 3 Níveis & 65,7 & 75,7 \\
\hline Acrílico & 47,2 & 30,56 \\
\hline Discectomia sem fusão e sem fixação & 39,35 & 36,51 \\
\hline Discectomia com fusão e sem fixação & 43,57 & 37,39 \\
\hline Discectomia com fusão e com fixação & 46,04 & 45,42 \\
\hline Placa/Cage Conjugados & 58,43 & 58,70 \\
\hline$\sum$ Via anterior & 42,79 & 41,40 \\
\hline
\end{tabular}

Entre as variáveis modalidades cirúrgicas por via anterior houve melhora estatisticamente significativa, $\operatorname{com} \alpha=0,001$, para todos os grupos e taxas de recuperação bastante significativas. Há tendência de melhora dos escores, com performance superior, no grupo onde foi praticado fusão e fixação (Tabela 11).

Observamos significativo déficits de fusão nos casos das corpectomias em mais de um nível, sendo muito significativa nos casos em três níveis (Tabela 12).

Em todas as modalidades pela via anterior o tratamento cirúrgico foi eficaz em diminuir a dor axial, porem no casos das discectomias sem fusão foi bem menos (Tabela 13).

Assim como ocorreu no déficit de fusão que é mais frequente nas corpectomias envolvendo mais de um nível vertebral os deslocamentos de enxerto também são mais frequemtes nestes e estão relacionados com o tamanho dos enxertos e as interfaces entre enxerto e vértebras (Tabela 14).

A cifotização ocorreu em $6,2 \%$ das vezes na via anterior, mais frequente nas discectomias que nas corpectomias (Tabela 15).

Houve diagnóstico de instabilidade pós cirúrgica em 5,6\% do total de casos em sua maior parte devido a problemas com enxertos e placas mal posicionados ou que migraram. Isto é evidenciado pela maior ocirrência nos casos com indução de fusão e fixação em relação aos sem (Tabela 16).

A síndrome do disco adjacente a fusão ocorreu em !6,87\% dos casos,sendo observada com mais freqüência em casos de discectomia com fusão e fixação, sendo mais freqüente nos casos de discectomias em 01 nível e menos quanto maior o número de níveis abordados.

Tabela 11. Modalidades cirúrgicas por via anterior e déficit de fusão intervertebral.

\begin{tabular}{c|c|c|c}
\hline & $\mathbf{n}$ & Não fusão & $\%$ \\
\hline Discectomia sem fusão e sem fixação & 56 & 10 & $17,85 \%$ \\
\hline Acrílico & 5 & 5 & $100 \%$ \\
\hline Nada & 51 & 5 & $9,8 \%$ \\
\hline Discectomia com fusão e sem fixação & 42 & 3 & $7,1 \%$ \\
\hline Discectomia com fusão e com fixação & 28 & 1 & $3,5 \%$ \\
\hline Corpectomia em 1 nível & 25 & 1 & $4 \%$ \\
\hline Corpectomia em 2 níveis & 7 & 1 & $14,2 \%$ \\
\hline Corpectomia em 3 níveis & 2 & 1 & $50 \%$ \\
\hline LVia anterior & 160 & 17 & $10,6 \%$ \\
\hline
\end{tabular}

Tabela 12. Modalidades cirúrgicas por via anterior e dor axial cervical, interescapular ou nos ombros, pós-operatória.

\begin{tabular}{c|c|c|c|c|c}
\hline & $\mathbf{n}$ & \multicolumn{2}{|c|}{$\begin{array}{c}\text { Dor axial } \\
\text { pré-operatória }\end{array}$} & \multicolumn{2}{c}{$\begin{array}{c}\text { Dor axial } \\
\text { pós-operatória }\end{array}$} \\
\hline Discectomia sem fusão e sem fixação & 56 & 33 & $59,9 \%$ & 28 & $50 \%$ \\
\hline Discectomia com fusão e sem fixação & 42 & 22 & $52,3 \%$ & 8 & $19,4 \%$ \\
\hline Discectomia com fusão e com fixação & 28 & 12 & $42,8 \%$ & 5 & $17,8 \%$ \\
\hline Corpectomia em 1 nível & 25 & 14 & $56 \%$ & 6 & $24 \%$ \\
\hline Corpectomia em 2 níveis & 7 & 2 & $28,5 \%$ & 1 & $14,2 \%$ \\
\hline Corpectomia em 3 níveis & 2 & 1 & $50 \%$ & 0 & 0 \\
\hline$\sum$ Via anterior & 160 & 103 & $64,3 \%$ & 30 & $18,7 \%$ \\
\hline
\end{tabular}

Tabela 13. Modalidades cirúrgicas por via anterior e deslocamento do enxerto.

\begin{tabular}{c|c|c|c}
\hline & $\mathbf{n}$ & Deslocamentodo enxerto & $\%$ \\
\hline Discectomia sem fusão e sem fixação & 56 & 4 - Acrílico & $7,1 \%$ \\
\hline Discectomia com fusão e sem fixação & 42 & 3 & $7,1 \%$ \\
\hline Discectomia com fusão e com fixação & 28 & 2 & $7,1 \%$ \\
\hline Corpectomia em 1 nível & 25 & 2 & $4 \%$ \\
\hline Corpectomia em 2 níveis & 7 & 1 & $14,2 \%$ \\
\hline Corpectomia em 3 níveis & 2 & 1 & $50 \%$ \\
\hline$\sum$ Via anterior & 160 & 13 & $8,1 \%$ \\
\hline
\end{tabular}

Tabela 14. Modalidades cirúrgicas por via anterior e cifotização pós-operatória.

\begin{tabular}{c|c|c|c}
\hline & $\mathbf{n}$ & Cifotização & $\%$ \\
\hline Discectomia sem fusão e sem fixação & 56 & 1 & $1,7 \%$ \\
\hline Discectomia com fusão e sem fixação & 42 & 3 & $7,1 \%$ \\
\hline Discectomia com fusão e com fixação & 28 & 5 & $6,2 \%$ \\
\hline Corpectomia em 1 nível & 25 & 1 & $4 \%$ \\
\hline Corpectomia em 2 níveis & 7 & 0 & 0 \\
\hline Corpectomia em 3 níveis & 2 & 0 & 0 \\
\hline$\sum$ Via anterior & 160 & 10 & $6,2 \%$ \\
\hline
\end{tabular}

Tabela 15. Modalidades cirúrgicas por via anterior e instabilidade pós-operatória.

\begin{tabular}{c|c|c|c}
\hline & $\mathbf{n}$ & Instabilidade & $\%$ \\
\hline Discectomia sem fusão e sem fixação & 56 & 2 & $3,5 \%$ \\
\hline Discectomia com fusão e sem fixação & 42 & 4 & $9,5 \%$ \\
\hline Discectomia com fusão e com fixação & 28 & 2 & $7,1 \%$ \\
\hline Corpectomia em 1 nível & 25 & 1 & $4 \%$ \\
\hline Corpectomia em 2 níveis & 7 & 0 & 0 \\
\hline Corpectomia em 3 níveis & 2 & 0 & 0 \\
\hline$\sum$ Via anterior & 160 & 9 & $5,6 \%$ \\
\hline
\end{tabular}

Tabela 16. Modalidades cirúrgicas por via anterior e síndrome do disco adjacente à fusão na coluna cervical.

\begin{tabular}{c|c|c|c}
\hline & $\mathbf{n}$ & Sd. disco adjacente & $\%$ \\
\hline Discectomia sem fusão e sem fixação & 56 & 10 & $17,85 \%$ \\
\hline Discectomia com fusão e sem fixação & 42 & 8 & $19,04 \%$ \\
\hline Discectomia com fusão e com fixação & 28 & 9 & $32,14 \%$ \\
\hline Corpectomia em 1 nível & 25 & 0 & 0 \\
\hline Corpectomia em 2 níveis & 7 & 0 & 0 \\
\hline Corpectomia em 3 níveis & 2 & 0 & 0 \\
\hline$\sum$ Via anterior & 160 & 27 & $16,87 \%$ \\
\hline
\end{tabular}

Via posterior (Tabelas 17 e 18)

Tabela 17. Modalidade cirúrgica por via posterior e evolução operatória JOA.

\begin{tabular}{c|c|c|c|c}
\hline & $\mathbf{f}$ & JOA pré & JOA pós & JOA tardio \\
\hline Laminoplastia & 16 & 10,37 & 12,56 & 12,93 \\
\hline Laminectomia & 18 & 9,38 & 11,16 & 10,77 \\
\hline Arcocristectomia & 6 & 10,5 & 11,5 & 11,83 \\
\hline$\sum$ Via posterior & 40 & 9,95 & 11,77 & 11,8 \\
\hline
\end{tabular}

$X-X^{1}=2,93 / X-X^{2}=3,3$

$X-X^{1}=1,53 / X-X^{2}=1,14$

$X-X^{1}=1,87 / X-X^{2}=2,87$

$X-X^{1}=2,14 / X-X^{2}=2,17$

$\mathrm{n}=16$ 2,93/2,48/4 = 2,93/0,62 = 4,72//// 3,3/2,48/4 = 3,3/0,62 = 5,32

$n=181,53 / 2,48 / 4,24=1,53 / 0,58=2,63 / / / / 1,14 / 2,48 / 4,24=1,14 / 0,58=1,96$

$\mathrm{n}=61,87 / 2,48 / 2,44=1,87 / 1,01=1,85 / / / / 2,87 / 2,48 / 2,44=2,87 / 1,01=2,84$ $n=402,14 / 2,48 / 6,32=2,14 / 0,39=5,48 / / / / 2,17 / 2,48 / 6,32=2,17 / 0,39=5,56$ nível de significância $\alpha=0,001$ e zcrítico 3,29 / $\delta=2,48 / \mathrm{X}=9,63$. 
Tabela 18. Modalidade cirúrgica por via posterior e evolução operatória segundo a Taxa de recuperação.

\begin{tabular}{c|c|c|c}
\hline & f & TX Rec Pre & TX Rec Tar \\
\hline Laminoplastia & 16 & 36,09 & 40,54 \\
\hline Laminectomia & 18 & 24,83 & 19,02 \\
\hline Arcocristectomia & 6 & 14,26 & 19,01 \\
\hline$\sum$ Via posterior & 40 & 27,75 & 27,73 \\
\hline
\end{tabular}

Entre as variáveis modalidades cirúrgicas por via posterior observamos melhora estatisticamente significativa para $\alpha=0,001$ no grupo das laminoplastias, melhora esta mantida, a longo prazo.

No grupo das arcocristectomias não há melhora estatisticamente significativa para $\alpha=0,001$ ou 0,05 ( $\mathrm{z}$ crítico $=2,58$ e 1,96) no período imediato, porém tardiamente há melhora significativa com $\alpha=0,01$ ( $z$ crítico $=2,58$ ). Também o n é baixo.

No grupo das laminectomias não há melhora significativa precoce com $\alpha=0,001$, porém para $\alpha=0,01$ ( $z$ crítico $=2,58$ ) há melhora significativamente estatística, porém tardiamente há piora do quadro com melhora estatística somente com $\alpha=0,05$ ( $\mathrm{z}$ crítico $=1,96)$ (Tabela 19).

Tabela 19. Idade e período de evolução pré-operatória.

\begin{tabular}{c|c|c|c}
\hline & f & Idade & Períod evol pré op \\
\hline Laminoplastia & 16 & 57,18 & 26,87 \\
\hline Laminectomia & 18 & 64,61 & 25,88 \\
\hline Arcocristectomia & 6 & 52,16 & 24,16 \\
\hline EVia posterior & 40 & 59,77 & 26,02 \\
\hline
\end{tabular}

\section{Intervalo de confiança.}

A média de idade no grupo das laminectomias está acima do limite superior do intervalo de confiaça para o grupo dos abordados pela via posterior, assim interferindo negativamente no desempenho de melhora do subgrupo (Tabela 20).

No s casos de laminoplastia não houve alteração na incidência de dor axial pós-operatório, não havendo melhora do sintoma também, porém sem piora, fato que ocorreu nas laminectomias e arcocristectomias (Tabela 21).

A cifotização ocorreu com freqüência muito grande nos casos submetidos a laminectomia e arcocristectomias e na mesma proporção que o ocorrido na via anterior em relação a laminoplastia (Tabela 22).

Ainda no caso das laminectomias houve incidência bastante significativa de diagnóstico de instabilidade pós-cirúrgica, fato não ocorrido na laminoplastia e arcocristectomia.

Tabela 20. Modalidades cirúrgicas por via posterior e dor axial pós-operatória.

\begin{tabular}{c|c|c|c|c|c}
\hline & $\mathbf{n}$ & \multicolumn{2}{|c|}{ Dor axial pré-operatória } & \multicolumn{2}{c}{ Dor axial pós-operatória } \\
\hline Laminoplastia & 16 & 9 & $56,2 \%$ & 9 & $56,2 \%$ \\
\hline Laminectomia & 18 & 10 & $55,5 \%$ & 15 & $83,3 \%$ \\
\hline Arcocristectomia & 6 & 3 & $50 \%$ & 4 & $66,6 \%$ \\
\hline$\sum$ Via anterior & 40 & 22 & $55 \%$ & 28 & $70 \%$ \\
\hline
\end{tabular}

Tabela 21. Modalidades cirúrgicas por via posterior e cifotização pós-operatória.

\begin{tabular}{c|c|c|c}
\hline & $\mathbf{f}$ & Cifotização & $\%$ \\
\hline Laminoplastia & 16 & 1 & $6,2 \%$ \\
\hline Laminectomia & 18 & 7 & $38,8 \%$ \\
\hline Arcocristectomia & 6 & 1 & $16,6 \%$ \\
\hline$\sum$ Via posterior & 40 & 9 & $22,5 \%$ \\
\hline
\end{tabular}

Tabela 22. Modalidades cirúrgicas por via posterior e instabilidade pós-operatória.

\begin{tabular}{c|c|c|c}
\hline & $\mathbf{f}$ & Instabilidade & $\%$ \\
\hline Laminoplastia & 16 & 0 & 0 \\
\hline Laminectomia & 18 & 2 & $11,1 \%$ \\
\hline Arcocristectomia & 6 & 0 & 0 \\
\hline$\sum$ Via posterior & 40 & 2 & $5 \%$ \\
\hline
\end{tabular}

Resultados comparativos entre a via anterior e a posterior (Figura 1)

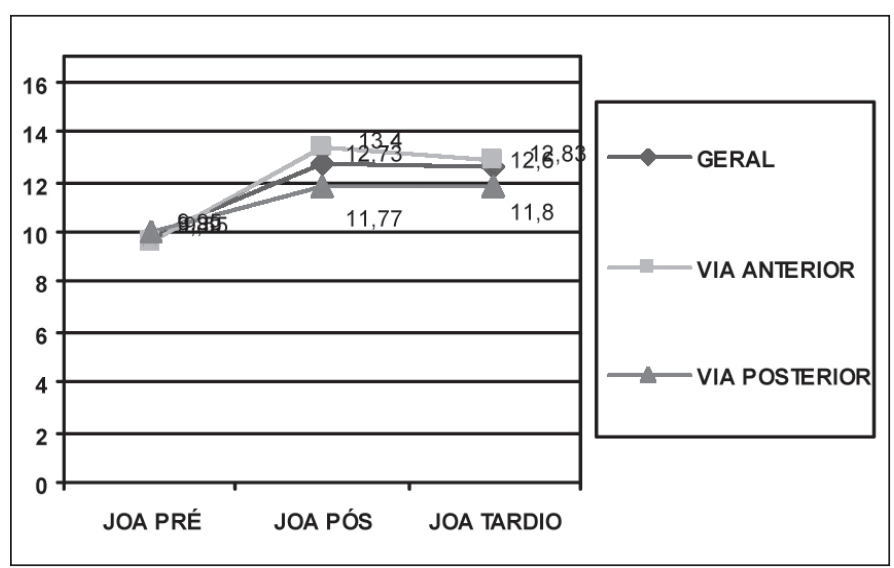

Figura 1. Evolução final em pontos de acordo com a escala da JOA.

A via anterior mostrou um desempenho de melhora neurológica, aferida em pontos pela escala da JOA ligeiramente superior à via posterior, ressalva para o fato da via posterior ter sido utilizada em um grupo com média de idade superior ao limite do intervalo de confiança esperado, Porém ambos apresentaram melhora estatisticamente significativa para $\alpha=0,001$ (Tabela 23).

No total as complicações relacionadas às vias de acesso foram significativamente superiores na via anterior, muitas relacionadas com as particularidades anatômicas da região como ocorrido com a lesão do nervo laringeu recorrente, lesão esofágica, utilização de enxerto e placas (Figura 2).

Tabela 23. Outras complicaçãoes vias anterior e posterior.

\begin{tabular}{c|c|c}
\hline Disfagia permanente & 3 & 0 \\
\hline Disfonia permanente & 4 & 0 \\
\hline Mau posicionamento de enxertos & 6 & 0 \\
\hline Mau posicionamento de placas & 5 & 0 \\
\hline Lesão de raiz & 5 & 1 \\
\hline Infecção sítio cirúrgico & 4 & 2 \\
\hline Pneumonia - sepsis & 3 & 2 \\
\hline$\sum$ Total & 30 & 5 \\
\hline
\end{tabular}

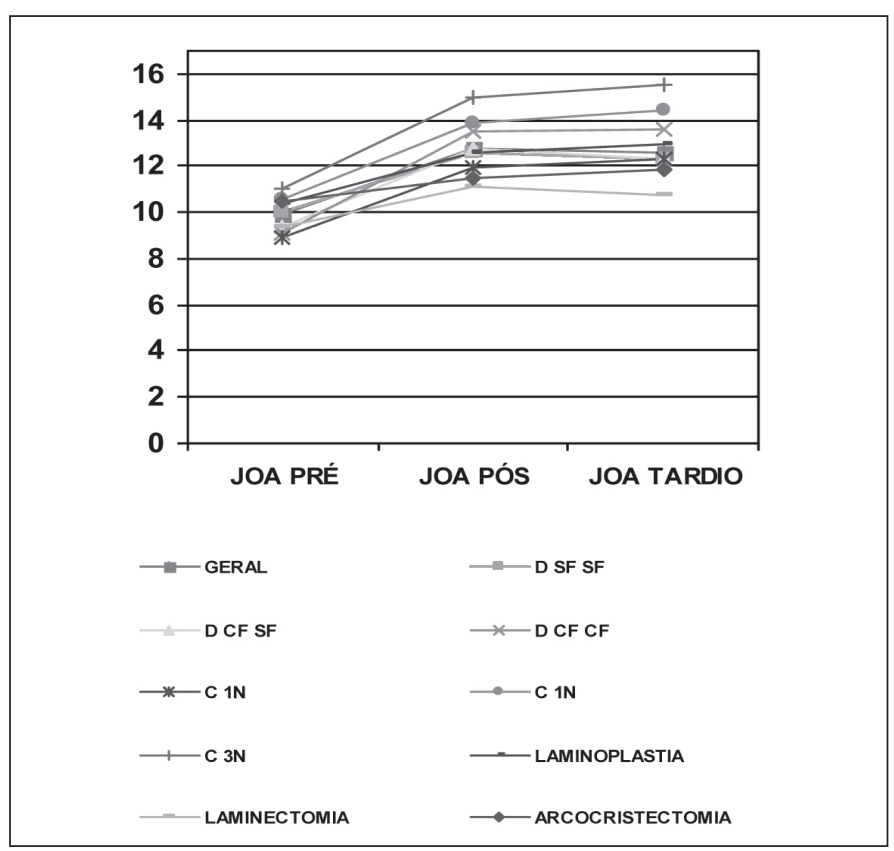

Figura 2. Modalidades cirúrgicas por via anterior e posterior e evolução operatória. 
Segmento pós-operatório (Tabela 24, Figura 3)

Tabela 24. Período de seguimento pós-operatório.

\begin{tabular}{c|c|c|c|c}
\hline & f & fr & Fr & $\%$ \\
\hline 24 & 29 & 0,145 & 0,145 & 14,5 \\
\hline $24+48$ & 79 & 0,395 & 0,54 & 39,5 \\
\hline $48+$ & 92 & 0,46 & 1,0 & 46 \\
\hline$\Sigma$ & 200 & 1,00 & 1,00 & 100 \\
\hline
\end{tabular}

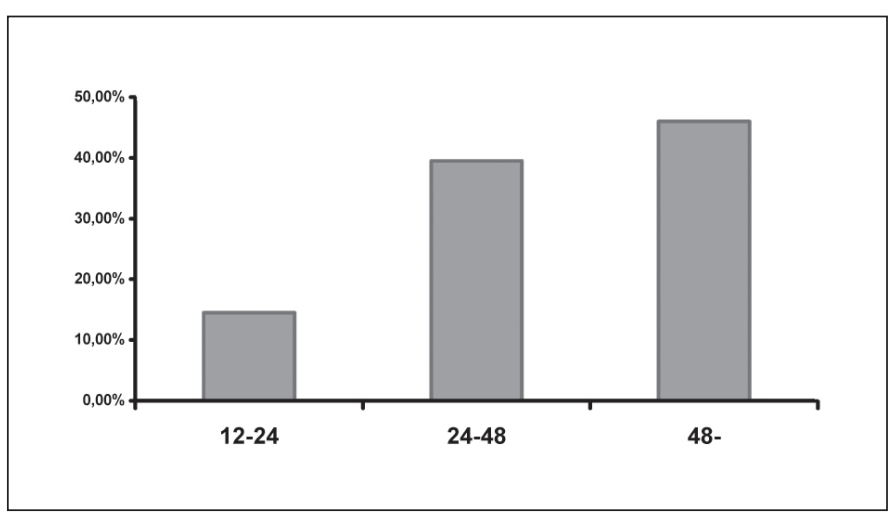

Figura 3. Distribuição percentual da média de seguimento pós-operatório.

O segmento mínimo para inclusão no trabalho foi de 24 meses. No total $46 \%$ dos casos tiveram segmento superior a 48 meses.

Degeneração discal adicional (Tabela 25)

Tabela 25. Escala de Kellgren pré-operatório e pós-operatório para os níveis adjacentes, via anterior e posterior.

\begin{tabular}{c|c|c|c|c}
\hline & Anterior & Posterior & Anterior & Posterior \\
\hline 0 & 65 & 0 & 30 & 0 \\
\hline 1 & 47 & 5 & 53 & 0 \\
\hline 2 & 35 & 17 & 50 & 19 \\
\hline 3 & 13 & 14 & 27 & 17 \\
\hline 4 & 0 & 4 & 0 & 4 \\
\hline Total & 160 & 40 & 160 & 40 \\
\hline
\end{tabular}

Houve piora ou progressão da degeneração discal em níveis adjacentes aos abordados cirurgicamente em $43,75 \%$ dos casos na via anterior (70 casos) e em 52,5\% (21 casos) na via posterior.

\section{Reabordagem}

Na via posterior em 02 casos houve necessidade de fixação com parafusos de massa lateral pelo desenvolvimento de instabilidade, em casos de laminectomia. Em outros dois casos, de laminectomias, a descompressão não se mostrou inadequada, após realização de RNM, e houve necessidade de ampliação da laminectomia. No caso da via anterior houve necessidade de re-intervenção em 04 casos para remoção do acrílico, em 04 houve necessidade por déficit de fusão, em 08 por deslocamento do enxerto, em 18 por motivos de síndrome do disco adjacente, e entre estes estão os que desenvolveram instabilidade. Entre as modalidades por via anterior em 04 casos de discectomia e interposição de acrílico, em 12 discectomia sem interposição de material qualquer, em 10 discectomia e fusão sem fixação, em 10 discectomia e fusão e fixação com placas e em 02 corpectomias (Tabela 26).

Tabela 26. Reabordagem.

\begin{tabular}{c|c|c|c}
\hline Anterior & 160 & 34 & $21,25 \%$ \\
\hline Posterior & 40 & 4 & $7,5 \%$ \\
\hline
\end{tabular}

\section{DISCUSSÃO}

Em um estudo publicado em 2010, Mary R. A. Cunningham ${ }^{31}$ comparou as opções técnicas para tratamento cirúrgico da mielopatia espondilótica cervical, por meio de revisão sistemática de estudos retrospectivos. Neste estudo foram comparados os resultados relativos à recuperação neurológica e complicações cirúrgicas. Inicialmente ela encontrou um total de 1735 artigos, sendo 11 incluídos na revisão, todos eles séries de casos retrospectivos. Quatro estudos comparando corpectomia multinível com laminoplastia, um estudo comparando laminectomia com laminoplastia, e dois comparando discectomia cervical anterior e fusão com laminoplastia. Em três estudos somente, o seguimento foi superior ha 10 anos.

A revisão concluiu que em todas as abordagens foram observados índices de recuperação neurológica similares. A laminoplastia teve um significante maior índice de dor cervical comparado à corpectomia multinível. A discectomia anterior com fusão mostrou aumento na chance de desenvolvimento de espondilose secundária adjacente, comparado com a lamioplastia. A corpectomia multinível e a laminectomia com fusão tiveram um significante maior índice de complicações, relacionados com o enxerto, instrumentação e via de acesso, além de significante diminuição na amplitude de movimento do pescoço, comparado à laminoplastia.

As principais publicações sobre resultados e técnicas para tratamento da mielorradiculopatia cervical são listados e resumidos abaixo:

Heller32, 2001, n=26, idade média 56 anos, mielopatia em múltiplos níveis, seguimento 26 meses (variação 2-9), laminoplastia $(n=13)$ e laminectomia com fusão $(n=13)$. Conclusão: Ambos apresentaram melhora na força, sensibilidade e dor.

Tani ${ }^{33}, 2002, n=26$, idede média 64 anos, OLLP, seguimento 50 meses (variação 7-93), descompressão micocirúrgica anterior com enxerto de ilíaco $(n=14)$ e laminoplastia $(n=12)$. Conclusão: Descompressão anterior apresentou melhores resultados funcionais. Houve piora neurológica em 33\% das laminoplastias e em zero no grupo da descompressão anterior.

Edwards ${ }^{12}, 2002$, EUA, retrospectivo, cohort, $n=26$, idade média 53 anos, mielopatia em múltiplos níveis, seguimento 44 meses (variação 16-101), laminoplastia em porta aberta $(n=3)$, com serra em $t(n=10)$ e corpectomia e fusão em múltiplos níveis $(n=13)$. Conclusão: Redução da mobilidade sagital em 57\% das corpectomias, e em $38 \%$ das laminoplastias. Utilização de menos analgésicos no grupo das laminoplastias

Kaminski34, 2004, n=42, idade média 54 anos, mielopatia em múltiplos níveis, segmento médio de 65 meses (variação 36-112), laminoplastia $(n=20)$ e laminectomia com fusão $(n=22)$. Conclusão: Melhora semelhante quanto a dor, marcha, força e sensibilidade em ambos os grupos. Laminoplastia apresentou menos complicações tardias.

Wada $^{35}, 2001$, Japão, retrospectivo, cohort, $n=47$, idade média 54 anos, mielopatia em múltiplos níveis, segmento de 13 meses, corpectomia $(n=23)$ e laminoplastia $(n=24)$. Conclusão: Dor axial $15 \%$ na corpectomia e $40 \%$ na laminoplastia, Mobilidade cervical redução de $51 \%$ na corpectomia e $71 \%$ na laminoplastia. O grupo da corpectomia apresentou maior tempo cirúrgico, maior perda sanguínea e taxa de pseudoartrose de $26 \%$.

Yonenobu ${ }^{36}$, 1992, Japan, retrospectivo, cohort, $n=83$, idade (54,3-56), mielopatia em múltiplos níveis, seguimento 43 a 53 meses, laminoplastia "open-door" com espaçador de titânio (42) e corpectomia (41). Conclusão: Melhora pontuação JOA em 4 pontos. Taxa de recuperação Laminoplastia 44,9 \pm 26,2 e corpectomia $53,3 \pm 30,2$. Em quatro casos de laminoplastia houve cifotização patológica.

Hosono $^{27}$, 1996, Japão, retrospectivo, cohort, $n=98$, idade (54,9-59,2), mielopatia em múltiplos níveis, seguimento corpectomia 52,6 meses (24-86) e laminoplastia 40,1 meses (24-102), corpectomia e laminoplastia porta aberta tipo dobradiça. Conclusão: melhora na pontuação JOA corpectomia 3,9 pontos e laminoplastia 4,5 pontos.

Dor axial pós operatória corpectomia 19\% e laminoplastia 60\%. 
Casha $^{37}, 2004, \mathrm{n}=28$, Idade média 54 anos, mielopatia em múltiplos níveis (61\%), tumor (32\%) e trauma (7\%), seguimento 15 meses (variação 4-48), laminoplastia com mini-placas de titânio. Conclusão: Melhora do diâmetro do canal em 3,6 mm. Em 70\% dos pacientes com MEC houve melhora neurológica.

Edwards $^{38}, 2000, n=18$, idade média54 anos, mielopatia em múltiplos níveis, seguimento de 24 meses (variação 18-36), laminoplastia com serra em t. Conclusão: Melhora pós-operatória na força $(78 \%)$, dor (83\%), parestesias (83\%). Melhora na escala de Nurick de 2,7 a 0,9.

Praharaj ${ }^{39}, 2000, n=24$, mielopatia espondilótica em múltiplos níveis (64\%) e OLLP (36\%), seguimento (1-14) meses, laminoplastia expansiva. Conclusão: Dor radicular melhorou em 75\%. Espasticidade foi reduzida em $88 \%$.

Lee $^{40}, 1997, n=25$, idade média de 60 anos, mielopatia espondilótica, seguimento de 18 a 43 meses, laminoplastia expansiva em porta aberta. Conclusão: Melhora na marcha (84\%). B\&B melhor em $77 \%$. Melhor ME/CV em 38\%.

Seichi ${ }^{41}, 2001$, Japan, $n=60$, idade média 55 anos, mielopatia espondilótica (58\%) e OLLP (42\%), seguimento de 154 meses (120218), laminoplastia em dupla porta. Conclusão: Melhora na escala da JOA em 4 pontos. Melhora neurológica em 91\%.

Takayasu ${ }^{42}, 2002, n=15$, idade média de 62,7 anos, mielopatia com estenose de canal, seguimento de 10,5 meses (variação 5-19), laminoplastia em dupla porta. Conclusão: Melhora na escala da JOA em 3,4 pontos. Sem perda do alinhamento sagital.

Tani $^{33,43}, 2002, n=30$, idade média 55 anos, estenose cervical em múltiplos níveis, seguimento médio 18 meses (6-53), laminoplastia com preservação dos elementos cervicais posteriores. Conclusão: Melhora neurológica em 97\% dos casos. Perda média de mobilidade de 7 graus.

Tomita ${ }^{44}, 1998, n=25$, idede média 57 anos, OLLP (52\%) e estenose congênita (48\%), seguimento 34 meses (24-48), laminoplastia com serra em t saw. Conclusão: Melhora na área do canal com expansão em 36\%. Melhora neurológica em 72\% dos casos.

Agrawal $^{45}, 2004, n=24$, idade média 57 anos, mielopatia Nurick III-V, seguimento 22 meses (6-46), laminoplastia em porta aberta de Hirabayashi, modificada com miniplacas de titânio. Conclusão: Melhora na escala de Nurick por $<3$ anos $=100 \%$, 3 a 6 anos $=$ $50 \%,>6$ anos $=0.96 \%$ bom ou regular pela escala de Odom.

Takami $^{46}, 2004, n=10$, idade média 62 anos, OLLP, seguimento 31 meses (8-52), laminoplastia tipo "lift-up". Conclusão: 13\% de redução na estenose. Melhora na função neurológica de 40-80\%.

Wang $^{47}, 2004, n=204$, idade média 63 anos, mielopatia com estenose de canal, seguimento de 16 meses, laminoplastia em porta aberta. Conclusão: $62 \%$ de melhora em 1 ou mais graus de Nurick.

Kumar $^{48}, 1999, n=25$, idade média 60 anos, mielopatia em múltiplos níveis, seguimento 48 meses (25-82), laminectomia e fusão/ fixação com placas de massa lateral. Conclusão: $80 \%$ de bons resultados. $76 \%$ melhoraram na escala de mielopatia.

Houten $^{49}, 2003, n=38$, idade média 65 anos, mielopatia espondilótica (76\%), OLLP (18\%) e ambos (5\%), seguimento 7 meses (2-18), laminectomia e placa cervical posterior. Conclusão: 97\% de melhora pela escala da JOA. Melhora da força muscular e função neurológica. Sem alteração no alinhamento sagital após 6 meses de seguimento.

Kaptain ${ }^{50}, 2000, n=46$, mielopatia em múltiplos níveis, seguimento de 48 meses, laminectomia. Conclusão: 29\% com melhora neurológica, 42\% sem alteração, 21\% de cifotização, a probabilidade de desenvolver deformidade é $2 x$ maior em colunas retificadas.

Schultz ${ }^{51}, 2000, n=72$, idade média 53 anos, mielopatia espondilótica (44\%), OLLP (8\%), trauma 26\% e cifose pós-laminectomia (20\%), seguiemnto 29 meses, descompressão e fusão, vias anterior e posterior combinadas. Conclusão: 100\% de fusão. Morbidade precoce $32 \%$. Morbidade tardia 5\%. Nenhuma re-operação.

Swank $^{52}, 1997, n=15$, idade média 56 anos, mielopatia em múltiplos níveis $(n=15)$, OLLP $(n=1)$ e trauma $(n=1)$, seguimento 30 meses, descompressão e fusão anterior, descompressão posterior e reconstrução com placas de massa lateral. Conclusão: Melhora neurológica em 87\%. Melhora do alinhamento sagital em 5 graus, 1 paciente com radiculopatia sensitiva

Isu ${ }^{53}, 1997, \mathrm{n}=40$, idade média 55 anos, OLLP, segmernto 36 meses (12-63), descompressão anterior e fusão com enxerto dos corpos vertebrais. Conclusão: 100\% de consolidação, 13\% com angulação anterior (cifotização). Sem complicações da área doadora.

Emery $^{9}, 1998, n=108$, idade média 58 anos, mielopatia espondilótica cervical, inclui OLLP, segmento 54 meses (24-202), Discectomia anterior, corpectomia parcial ou corpectomia subtotal com enxerto autólogo. Conclusão: Melhora na escala de Nurick de 1,2 a 2,4. 1 caso com pseudoartrose (3\%) e mau resultado.

Wang ${ }^{54}, 2006$, China, $n=42$, idade média 57,2 anos (46-72), mielopatia multinível, segmento 42,6 meses (30-54), discectomia cervical anterior e fusão em múltiplos níveis e laminoplastia expansiva em dupla porta. Conclusão: melhora pontuação JOA em 04 pontos e taxa de recuperação discectomia 60,8 \pm 20,7\% e laminoplastia 57,1 1 16,4\%. Fusão em todos os casos e sem cifotização.

Kawakami13, 2000, Japão, n=63, idade média $(51,1$ e 61,5), mielopatia com estenose em 1 ou 2 níveis: discectomia cervical anterior e fusão (9), mielopatia com estenose em 03 níveis ou mais (54): Iaminoplastia, seguimento 68 meses, laminoplastia expansiva com reposicionamento do processo espinhoso. Conclusão: Taxa de recuperação discectomia 49,0\% e laminoplastia 58,6\%, taxa de fusão 100\%, sem alterações no alinhamento sagital pós-operaytório.

Kawaguchi ${ }^{5}$, 2003, Japan, n=35, mielopatia em múltiplos níveis, laminoplastia em porta aberta modificada em bloco, seguimento de 158 meses. Conclusão: melhora significativa estatisticamente na pontuação na escala da JOA. Redução em 31,9\% na amplitude de movimento cervical e aumento na dor axial cervical em 33\%.

Ikenaga $^{56}, 2006$, Japan, $n=12$, idade média, mielopatia em múltiplos níveis, corpectomia cervical anterior e fusão em múltiplos níveis, seguimento de 120 a 168 meses. Conclusão: Aumento em 6,5\% na dor cervical axial de forma leve. Em um caso piora na destreza manual e um caso de não fusão e dois casos de lesão radicular.

Em nosso estudo a análise das médias obtidas com a pontuação segundo a escala da JOA e com a taxa de recuperação observamos incremento estatisticamente significativo $(\alpha=0,001)$ nas médias pós-operatórias, precoce e tardiamente, entre os grupos operados por via anterior e posterior, concluindo que houve melhora estatisticamente significativa para os dois grupos entre os períodos pré e pós-operatório.

Observa-se que a média de idade do grupo onde a cirurgia se deu por via posterior está acima do limite superior do intervalo de confiança, implicando em um envolvimento de outro fator preditivo de mau prognóstico.

Entre as modalidades cirúrgicas, em todas as praticadas pela via anterior há evidências estatísticas $(\alpha=0,001)$ de melhora, evidenciado pelo incremento nas médias de pontuação obtidos pela escala da JOA e índice de recuperação, com exceção de corpectomia em 2 níveis, onde o grupo apresentava média de idade superior ao limite superior do intervalo de confiança e $n$ pequeno, porém com recuperação tardia.

Entre as modalidades cirúrgicas praticadas pela via posterior observamos melhora estatisticamente significativa $(\alpha=0,001)$ somente no grupo das laminoplastias, melhora esta mantida, a longo prazo. No grupo das arcocristectomias o número de casos é muito baixo, prejudicando a análise.

No grupo das laminectomias não observamos melhora significativa $(\alpha=0,001)$, precoce e tardiamente. Observamos que houve piora tardia das médias obtidas pela escala da JOA, indicando que há piora neurológica tardiamente, que pode estar associada à ocorrência de complicações tardias com cifotização e instabilidade.

Portanto, tanto a via anterior quanto a posterior foram eficazes em produzir melhora ou incremento neurológico pós-operatório, sendo que as médias da via posterior são inferiores às da anterior em virtude dos resultados ruins obtidos com a laminectomia, porém a laminoplastia tem resultados semelhantes aos da via anterior.

Quanto à laminectomia sem fixação não houve produção da 
melhora esperada, ocorrendo ainda piora neurológica tardia.

No total, $10,6 \%$ dos casos abordados por via anterior apresentaram déficit de fusão intervertebral, pós-cirúrgica, mais freqüente nas corpectomias e nas discectomias sem indução de fusão. Nos casos com interposição de acrílico a não fusão já era esperada.

Em 8,1\% dos casos, pela via anterior, houve deslocamento do enxerto, mais comum nas discectomias em relação às corpectomias, diferente do que mostra a literatura.

Em $16,87 \%$ dos casos submetidos à fusão intervertebral foi diagnosticada a síndrome do disco adjacente á fusão, sendo que nos casos não submetidos à fusão, não houve ocorrência clínica e radilógica similar.

Outras complicações como disfonia, disfagia, lesão de raiz, mau posicionamento de enxerto, mau posicionamento de placas somaram $14,3 \%$ de complicações, atribuídas a via anterior.

No total contabilizamos $49,87 \%$ de complicações atribuídas somente à via anterior, ou seja, superior à via posterior.

Com relação à dor axial na via anterior houve melhora em 45,6\% dos sintomas pré-operatórios enquanto na via posterior houve piora em 15\% nos sintomas pós-operatórios, mais importante no caso das laminectomias, enquanto que nas laminoplastias observamos a manutenção das freqüências observadas no período pré-operatório.

Em relação à cifotização pós-operatória observamos ocorrência do achado em $22,5 \%$ dos casos na via posterior, com $38,8 \%$ nas laminectomias e 6,2\% nas laminoplastias, e em 6,2\% na via anterior, mostrando uma predominante maior freqüência de ocorrência no acesso posterior com laminectomia, mas não na laminoplastia.

Instabilidade, segundo os critérios de White e Panjabi, e não com desvio em cifose, pós-cirúrgica foi observada em 5,6\% na via anterior e em $5 \%$ na via posterior, mostrando uma equivalência entre as duas vias.

Houve necessidade de re-abordagem cirúrgica em 21,25\% dos casos abordados pela via anterior e em 7,5\% dos casos abordados pela posterior, mostrando ampla superioridade na via anterior.

Quanto à degeneração discal adicional foi observado em $43,75 \%$ na via anterior e em 52,5\% na via posterior, sem correlação com o desenvolvimento de novos quadros de mielorradiculopatia ou a síndrome do disco adjacente à fusão, o que se correlaciona com a gravidade de degenerações pré-existentes e adjacentes a segmentos fundidos, na via anterior.

\section{CONCLUSÃO}

O estudo evidencia que as vias, anterior e posterior foram eficazes em produzir melhora neurológica pós-operatória, evidenciado por incremento na pontuação pós-operatória segundo a escala da JOA e a taxa de recuperação. Na via anterior as pontuações e a taxa de recuperação foram superiores às observadas na posterior. A laminectomia sem fusão não produziu melhora estatisticamente significativa e produziu piora neurológica tardia.

A via anterior teve um significante maior índice de complicações, relacionados a déficit de fusão intervertebral, deslocamento de enxerto, síndrome de disco adjacente, disfonia, disfagia, o mau posicionamento de enxerto e placas, lesão de raiz nervosa e significante maior índice de re-intervenção cirúrgica

Na via posterior observou-se uma significante maior ocorrência de instabilidade em cifose pós-operatória na laminectomia, não sendo observado na laminoplastia que teve índices semelhantes aos encontrados nas discectomias e nas corpectomias.

A laminoplastia não evidenciou melhora no sintoma de dor axial pós-operatória, a laminectomia evidenciou piora significativa enquanto que nas discectomias e corpectomias houve significativa melhora do sintoma.

\section{REFERÊNCIAS}

1. Baron EM, Young WF. Cervical spondylotic myelopathy: a brief review of its pathophysiology, clinical course, and diagnosis. Neurosurgery. 2007 ;60(1Supp1 1):S35-41.

2. Symon L, Lavender P. The surgical treatment of cervical spondylotic myelopathy. Neurology. 1967;17(2):117-27.

3. Jumah KB, Nyame PK. Relationship between load carrying on the head and cervical spondylosis in Ghanaians. West Afr J Med. 1994;13(3):181-2.

4. Crandall PH, Batzdorf U. Cervical spondylotic myelopathy. J Neurosurg. 1966;25(1):57-66.

5. Emery SE. Cervical spondylotic myelopathy: diagnosis and treatment. J Am Acad Orthop Surg. 2001;9(6):376-88

6. Epstein JA, Epstein NE. The surgical management of cervical spinal stenosis, spondylosis, and myeloradiculopathy by means of the posterior approach. In: Sherk HH, Dunn EJ, Eismont FJ, et al., editors. The cervical spine, 2nd ed. Philadelphia: J.B. Lippincott; 1989. p. 625-43.

7. McCormick WE, Steinmetz MP, Benzel EC. Cervical spondylotic myelopathy: make the difficult diagnosis, then refer for surgery. Cleve Clin J Med. 2003;70(10):899-904.

8. Witwer BP, Trost GR. Cervical spondylosis: ventral or dorsal surgery. Neurosurgery. 2007;60(1 Supp1 1):S130-6

9. Emery SE, Bohlman HH, Bolesta MJ, Jones PK. Anterior cervical decompression and arthrodesis for the treatment of cervical spondylotic myelopathy. Two to seventeen-year follow-up. J Bone Joint Surg Am. 1998;80(7):941-51.

10. Bohlman HH, Emery SE, Goodfellow DB, Jones PK. Robinson anterior cervical discectomy and arthrodesis for cervical radiculopathy. Long-term follow-up of one hundred and twenty-two patients. J Bone Joint Surg Am. 1993;75(9):1298-307.

11. DiAngelo DJ, Foley KT, Vossel KA, Rampersaud YR, Jansen TH. Anterior cervical plating reverses load transfer through multilevel strut-grafts. Spine (Phila Pa 1976). 2000;25(7):783-95.

12. Edwards $\mathrm{CC}$ 2nd, Heller JG, Murakami H. Corpectomy versus laminoplasty for multilevel cervical myelopathy: an independent matched-cohort analysis. Spine (Phila Pa 1976). 2002 Jun 1;27(11):1168-75

13. Kawakami M, Tamaki T, Iwasaki H, Yoshida M, Ando M, Yamada H. A comparative study of surgical approaches for cervical compressive myelopathy. Clin Orthop Relat Res. 2000;(381):129-36.

14. Emery SE, Fisher JR, Bohlman HH. Three-level anterior cervical discectomy and fusion: radiographic and clinical results. Spine (Phila Pa 1976). 1997;22(22):2622-4.

15. Chiba $K$, Toyama $Y$, Watanabe M, Maruiwa H, Matsumoto M, Hirabayashi K. Impact of

longitudinal distance of the cervical spine on the results of expansive open-door laminoplasty. Spine (Phila Pa 1976). 2000;25(22):2893-8

16. Tanaka J, Seki N, Tokimura F, Doi K, Inoue S. Operative results of canal-expansive laminoplasty for cervical spondylotic myelopathy in elderly patients. Spine (Phila Pa 1976) 1999:24(22):2308-12.

17. Yonenobu K, Okada K, Fuji T, Fujiwara K, Yamashita K, Ono K. Causes of neurologic deterioration following surgical treatment of cervical myelopathy. Spine (Phila Pa 1976). 1986;11(8):818-23

18. Pal GP, Routal RV. The role of the vertebral laminae in the stability of the cervical spine. J Anat. 1996;188 ( Pt 2):485-9.

19. Yonenobu K, Hosono N, Iwasaki M, Asano M, Ono K. Neurologic complications of surgery for cervical compression myelopathy. Spine (Phila Pa 1976). 1991;16(11):1277-82

20. Brodke DS, ZdeblickTA. Modified Smith-Robinson procedure for anterior cervical discectomy and fusion. Spine (Phila Pa 1976). 1992;17(10 Suppl):S427-30

21. Bernard TN Jr, Whitecloud TS 3rd. Cervical spondylotic myelopathy andmyeloradiculopathy. Anterior decompression and stabilization with autogenous fibula strut graft. Clin Orthop Relat Res. 1987:(221):149-60.

22. Vaccaro AR, Falatyn SP, Scuderi GJ, Eismont FJ, McGuire RA, Singh K, et al.. Early failure of long segment anterior cervical plate fixation. J Spinal Disord. 1998;11(5):410-5

23. Higashi H, Yabuki S, Hayabara T, Ikeda H. Studies on pathogenesis of cervical spondylotic myelopathy: sagittal diameter of the spinal canal and cerebrospinal fluid characteristics in cervical spondylosis. Folia Psychiatr Neurol Jpn. 1974;28(1):35-44.

24. Sim FH, Svien HJ, Bickel WH, Janes JM. Swan-neck deformity following extensive cervica laminectomy. A review of twenty-one cases. J Bone Joint Surg Am. 1974;56(3):564-80.

25. Cusick JF, Pintar FA, Yoganandan N. Biomechanical alterations induced by multileve cervical laminectomy. Spine (Phila Pa 1976). 1995 Nov;20(22):2392-8.

26. Yasuoka S, Peterson HA, MacCarty CS. Incidence of spinal column deformity after multilevel laminectomy in children and adults. J Neurosurg. 1982;57(4):441-5.

27. Hosono N, Yonenobu K, Ono K. Neck and shoulder pain after laminoplasty. A noticeable complication. Spine (Phila Pa 1976). 1996;21(17):1969-73.

28. Yoshida M, Tamaki T, Kawakami M, Nakatani N, Ando M, Yamada H, et al. Does reconstruction of posterior ligamentous complex with extensor musculature decrease axial symptoms after cervical laminoplasty? Spine (Phila Pa 1976). 2002 ;27(13):1414-8.

29. Meluzzi A, Taricco MA, Guirado VMP, Pena Dias MR, Nakaguawa G, Jacobsen T M: Síndrome do disco adjacente à fusão (Síndrome de Transição) na coluna cervical: resultados 
segundo critérios clínicos e radiológicos. Coluna/Columna. 2010;9(1):49-57

30. Kellgren JH, Jeffrey MR, Ball J, editors. The epidemiology of chronic rheumatism. Atlas of standard radiographs. Oxford: Blackwell Scientific; 1963. p.14-9.

31. Cunningham MR, Hershman S, Bendo J. Systematic review of cohort studies comparing surgical treatments for cervical spondylotic myelopathy. Spine (Phila Pa 1976). 2010;35(5):537-43

32. Heller JG, Edwards CC, 2nd, Murakami H and Rodts GE: Laminoplasty versus laminectomy and fusion for multilevel cervical myelopathy: an independent matched cohort analysis. Spine. 26: 1330-6, 2001

33. Tani T, Ushida T, Ishida K, lai H, Noguchi T and Yamamoto H: Relative safety of anterior microsurgical decompression versus laminoplasty for cervical myelopathy with a massive ossified posterior longitudinal ligament. Spine. 27: 2491-8, 2002.

34. Kaminsky SB, Clark CR and Traynelis VC: Operative treatment of cervical spondylotic myelopathy and radiculopathy. A comparison of laminectomy and laminoplasty at five year average follow-up. lowa Orthop J. 24: 95-105, 2004.

35. Wada E, Suzuki S, Kanazawa A, Matsuoka T, Miyamoto S and Yonenobu K: Subtotal corpectomy versus laminoplasty for multilevel cervical spondylotic myelopathy: a long-term follow-up study over 10 years. Spine. 26: 1443-7, 2001

36. Yonenobu K, Fuji T, Ono K, ET al. Choice of surgical treatment for multi-segmental cervical spondylotic myelopathy, Spine, 1992;10:710-6.

37. Casha S, Engelbrecht HA, DuPlessis SJ and Hurlbert RJ: Suspended laminoplasty for wide posterior cervical decompression and intradural access: results, advantages, and complications. J Neurosurg Spine. 1: 80-6, 2004.

38. Edwards CC, 2nd, Heller JG and Silcox DH, 3rd: T-Saw laminoplasty for the management of cervical spondylotic myelopathy: clinical and radiographic outcome. Spine. 25 $1788-94,2000$.

39. Praharaj SS, Vasudev MK and Kolluri VR: Laminoplasty: an evaluation of 24 cases. Neurol India. 48: 249-54, 2000.

40. Lee TT, Manzano GR and Green BA: Modified open-door cervical expansive laminoplasty for spondylotic myelopathy: operative technique, outcome, and predictors for gait improvement. J Neurosurg. 86: 64-8, 1997.

41. Seichi A, Takeshita K, Ohishi I, Kawaguchi H, Akune T, Anamizu Y, Kitagawa T and Nakamura K: Long-term results of double-door laminoplasty for cervical stenotic myelopathy. Spine. 26: 479-87, 2001.

42. Takayasu M, Takagi T, Nishizawa T, Osuka K, NakajimaT and Yoshida J: Bilateral open-door cervical expansive laminoplasty with hydroxyapatite spacers and titanium screws. J Neurosurg Spine. 96: 22-8, 2002.

43. Tani S, Isoshima A, Nagashima Y, Tomohiko Numoto R and Abe T: Laminoplasty with preservation of posterior cervical elements: surgical technique. Neurosurgery. 50: $97-$ 101: discussion 101-2, 2002.

44. Tomita K, Kawahara N, Toribatake Y and Heller JG: Expansive midline T-saw laminoplasty (modified spinous process-splitting) for the management of cervical myelopathy. Spine. 23: 32-7, 1998.

45. Agrawal D, Sharma BS, Gupta A and Mehta VS: Efficacy and results of expansive laminoplasty in patients with severe cervical myelopathy due to cervical canal stenosis. Neurol India. 52: 54-8, 2004.

46. Takami T, Ohata K, Goto T, Nishikawa M, Nishio A, Tsuyuguchi N and Hara M: Lift-up laminoplasty for myelopathy caused by ossification of the posterior longitudinal ligament of the cervical spine. Neurol India. 52: 59-63, 2004.

47. Wang MY, Shah $S$ and Green BA: Clinical outcomes following cervical laminoplasty for 204 patients with cervical spondylotic myelopathy. Surg Neurol. 62: 487-92, 2004.

48. Kumar VG, Rea GL, Mervis LJ and McGregor JM: Cervical spondylotic myelopathy: functional and radiographic long-term outcome after laminectomy and posterior fusion. Neurosurgery. 44: 771-7; discussion 777-8, 1999.

49. Houten JK and Cooper PR: Laminectomy and posterior cervical plating for multileve cervical spondylotic myelopathy and ossification of the posterior longitudinal ligament: effects on cervical alignment, spinal cord compression, and neurological outcome. Neurosurgery. 52: 1081-7; discussion 1087-8, 2003

50. Kaptain GJ, Simmons NE, Replogle RE and Pobereskin L: Incidence and outcome of kyphotic deformity following laminectomy for cervical spondylotic myelopathy. J Neurosurg. 93: 199-204, 2000

51. Schultz KD, Jr., McLaughlin MR, Haid RW, Jr., Comey CH, Rodts GE, Jr. and Alexander $\mathrm{J}$ : Single-stage anterior-posterior decompression and stabilization for complex cervical spine disorders. J Neurosurg Spine. 93: 214-21, 2000.

52. Swank $M L$, Sutterlin $C E, 3 r d$, Bossons $C R$ and Dials $B E$ : Rigid internal fixation with lateral mass plates in multilevel anterior and posterior reconstruction of the cervical spine. Spine. 22: 274-82, 1997.

53. Isu T, Minoshima $\mathrm{S}$ and Mabuchi S: Anterior decompression and fusion using bone grafts obtained from cervical vertebral bodies for ossification of the posterior longitudinal ligament of the cervical spine: technical note. Neurosurgery. 40: 866-9; discussion 869-70, 1997.

54. Wang B, Liu H, Wang H, et al. Segmental instability in cervical spondylotic myelopathy with severe disc degeneration, Spine 2006;31:1327-31.

55. Kawagushi $\mathrm{Y}$, Kanamori $\mathrm{M}$, Ishihara $\mathrm{H}$, et al. Minimum 10-year follow-up after en bloc cervical laminoplasty. Clin Orthop Relat Res 2003;411:129-39.

56. Ikenaga M, Shikata J, Tanaka C. Long-term results over 10 years of anterior corpectomy and fusion for multilevel cervical myelopathy. Spaine 2006:31:1568-74; discussion 75. 